\title{
ON THE FOURIER TRANSFORM ON THE INFINITE SYMMETRIC GROUP
}

\author{
A. M. Vershik* and N. V. Tsilevich*
}

UDC 517.986

\begin{abstract}
We present a sketch of the Fourier theory on the infinite symmetric group $\mathfrak{S}_{\infty}$. As a dual space to $\mathfrak{S}_{\infty}$, we suggest the space (groupoid) of Young bitableaux $\mathcal{B}$. The Fourier transform of a function on the infinite symmetric group is a martingale with respect to the so-called full Plancherel measure on the groupoid of bitableaux. The Plancherel formula determines an isometry of the space $l^{2}\left(\mathfrak{S}_{\infty}, m\right)$ of square summable functions on the infinite symmetric group with the counting measure and the space $L^{2}(\mathcal{B}, \tilde{\mu})$ of square integrable functions on the groupoid of bitableaux with the full Plancherel measure. Bibliography: 16 titles.
\end{abstract}

\section{INTRODUCTION}

The duality between spaces of functions on a group and spaces of some objects on the set of irreducible representations of this group has always been attracting particular attention of specialists in representation theory and analysis, and there are hundreds of papers on this subject. In the case of commutative groups, there is a well-developed canonical theory, which is a direct generalization of the theory of classical Fourier series and integrals; it uses the theory of characters of commutative locally compact groups.

In the case of noncommutative groups, where irreducible representations are no longer necessarily onedimensional, there is no indisputable and generally accepted candidate to the role of Fourier theory, and there are many different versions of such a theory. Some facts and notions similar to the commutative case, such as Plancherel measure and Plancherel formula, Bochner theorem, central functions, etc., are well studied and widely used, but they are far from exhausting the problem. The point of view according to which the Fourier transforms of functions on a group are operator-valued functions on the set of irreducible representations (or sections of some tautological foliations over this set) is a too literal generalization of the commutative case and does not lead to any interesting results in particular cases.

In this paper, we present a sketch of a generalization of the Fourier theory for the infinite symmetric group. Our approach is based on the fact that this group has an inductive (increasing) family of subgroups, which leads to the appearance of many additional structures, such as the Gelfand-Tsetlin algebra, Young diagrams and tableaux, etc. Undoubtedly, a similar theory can be developed for other inductive families of groups, but here we restrict ourselves only to the symmetric groups.

We do not reproduce well-known definitions, which can be found in the textbooks and papers mentioned in the list of references. However, relatively new notions developed in recent years are described in more detail. Since this article will be continued in subsequent papers on the Fourier transform on the symmetric groups and other classical series of groups, we do not try to give all relevant references and describe all arising links, but restrict ourselves only to necessary definitions. However, we would like to mention that this series of papers naturally generalizes and exploits the series of papers by the first author and S. V. Kerov (see, e.g., [13]) started about 30 years ago and devoted to asymptotic representation theory and the inductive approach to this theory, as well as subsequent papers on close subjects by G. I. Olshanski and others (see, e.g., [8, 7]).

As a dual space to the symmetric group, we suggest the space (groupoid) of Young bitableaux, i.e., the space of pairs of tableaux with the same diagram. In fact, it is the space of matrix elements of all irreducible representations in a special basis, namely, the Gelfand-Tsetlin basis. Thus the Fourier transform of a function on the symmetric group is, by definition, a function on bitableaux; and instead of matrix-valued functions on the space of irreducible representations, we consider scalar functions which are the matrix elements of these representations in the chosen basis. The groupoid of bitableaux is equipped with the so-called full Plancherel measure, and the Plancherel formula determines an isometry of two Hilbert spaces of square integrable functions: the $L^{2}$ space on the symmetric group with the Haar measure, and the $L^{2}$ space on the groupoid of bitableaux with the full Plancherel measure.

Remarkably, these statements can be extended to the infinite symmetric group. Note that the space of classes of irreducible representations of this group is immense, so that the ordinary (operator-valued) version of duality

*St.Petersburg Department of the Steklov Mathematical Institute, St.Petersburg, Russia, e-mail: vershik@pdmi.ras.ru, natalia@pdmi.ras.ru.

Translated from Zapiski Nauchnykh Seminarov POMI, Vol. 325, 2005, pp. 61-82. Original article submitted May 25, 2005. 
cannot even be formulated. However, the suggested approach allows us to consider the problem of describing the Fourier transforms of different classes of functions on the group, etc. The crucial role is played by the Gelfand-Tsetlin maximal commutative subalgebra, which diagonalizes in all representations.

In the more general context of inductive limits of groups and algebras, as well as in the case of symmetric groups, we pay special attention to projective limits of groups and algebras with respect to some conditional expectations (not necessarily positive). For the symmetric groups, a nontrivial example of such an expectation and projective limit was first considered in $[4,5]$ (virtual permutations). In Fourier theory, projective limits lead to martingale theory on noncommutative groupoids, which, apparently, was not studied earlier.

\section{SymmetriC GROUPS $\mathfrak{S}_{n}$}

The classical representation theory of the finite symmetric groups is described, for instance, in the books [2, 1]; these books also contain references to other sources on this subject. In [15] (see also [16]), a new approach to this theory is presented, in which Young diagrams and tableaux and the branching rule for irreducible representations appear in a natural way; this approach is based on the systematic use of the Gelfand-Tsetlin algebras and Young-Jucys-Murphy elements. The classical (operator-valued) theory of Fourier transform on finite groups can be found, e.g., in [3]. Note that there is also a remarkable bijection ("combinatorial Fourier transform"), called the Robinson-Schensted-Knuth (RSK) correspondence, between the elements of the symmetric group and the bitableaux; see [11].

\subsection{Basic definitions and notation}

Let $\mathfrak{S}_{n}$ be the symmetric group of degree $n, \mathbb{C}\left[\mathfrak{S}_{n}\right]$ be the group algebra of $\mathfrak{S}_{n}$, and $m_{n}$ be the counting measure on $\mathfrak{S}_{n}$ (that is, $m_{n}(w)=1$ for every $\left.w \in \mathfrak{S}_{n}\right)$.

Denote by $\mathbb{Y}_{n}$ the set of Young diagrams with $n$ cells (by definition, $\mathbb{Y}_{0}$ consists of the empty diagram $\emptyset$ ). This set parametrizes the irreducible representations of the group $\mathfrak{S}_{n}$. Denote by $\pi_{\lambda}$ the irreducible unitary representation of $\mathfrak{S}_{n}$ corresponding to a digram $\lambda \in \mathbb{Y}_{n}$, by $\chi_{\lambda}$ the character of $\pi_{\lambda}$, and by $\operatorname{dim} \lambda$ the dimension of $\pi_{\lambda}$.

The branching graph of the irreducible representations of the symmetric groups is the Young graph $\mathbb{Y}$ defined as follows: the vertex set of $\mathbb{Y}$ is $\cup_{n} \mathbb{Y}_{n}$, and two vertices $\lambda \in \mathbb{Y}_{n}$ and $\mu \in \mathbb{Y}_{n-1}$ are joined by an edge whenever $\mu \subset \lambda$.

Denote by $T(\lambda)$ the set of Young tableaux of shape $\lambda \in \mathbb{Y}_{n}$, i.e., the set of paths in the Young graph from $\emptyset$ to $\lambda$. This set consists of $\operatorname{dim} \lambda$ elements. Given a tableau $t \in T(\lambda)$, set $\operatorname{dim} t=\operatorname{dim} \lambda$. Let $T_{n}=\cup_{\lambda \in \mathbb{Y}_{n}} T(\lambda)$ be the set of Young tableaux with $n$ cells, i.e., the set of paths of length $n$ in the Young graph.

According to the branching rule for irreducible representations of the symmetric groups, with each Young tableau $t \in T(\lambda)$ one can associate a one-dimensional subspace in the space $V_{\lambda}$ of the representation $\pi_{\lambda}$. Choose a unit vector $h_{t}$ in this subspace. The obtained basis $\left\{h_{t} ; t \in T(\lambda)\right\}$ in the space $V_{\lambda}$ is called the Gelfand-Tsetlin basis.

Let $B_{n}=\left\{(s, t): s, t \in T_{n}\right.$ are of the same shape $\}$ be the space of bitableaux (loops) of size $n$, i.e., the set of pairs of paths of length $n$ in the Young graph ending at the same vertex. Clearly, $\operatorname{dim} s=\operatorname{dim} t$ whenever $(s, t) \in B_{n}$.

\subsection{The Plancherel measure}

There are three closely related measures - on the space of Young diagrams, on the space of Young tableaux, and on the space of Young bitableaux, which will be called Plancherel measures.

The most classical of these measures is the Plancherel measure on the space $\mathbb{Y}_{n}$ of Young diagrams with $n$ cells, i.e., on the space of irreducible representations of the group $\mathfrak{S}_{n}$; it is given by the formula

$$
\operatorname{Pl}_{n}(\{\lambda\})=\frac{\operatorname{dim}^{2} \lambda}{n !}, \quad \lambda \in \mathbb{Y}_{n}
$$

It is a probability measure, as follows from the classical Burnside formula:

$$
\sum_{\lambda \in \mathbb{Y}_{n}} \frac{\operatorname{dim}^{2} \lambda}{n !}=1 .
$$

Now consider the measure on the space $T_{n}$ of Young tableaux with $n$ cells whose projection to $\mathbb{Y}_{n}$ coincides with the Plancherel measure $\mathrm{Pl}_{n}$ and, given $\lambda \in \mathbb{Y}_{n}$, the distribution on the set $T(\lambda)$ of tableaux of shape $\lambda$ is 
uniform; this measure is called the Plancherel measure on the space of Young tableaux, and it will be denoted by the same symbol; obviously, it is also a probability measure, and

$$
\mathrm{Pl}_{n}(\{t\})=\frac{\operatorname{dim} t}{n !}, \quad t \in T_{n} .
$$

Finally, the Plancherel measure on the space of bitableaux $B_{n}$ is given by the formula

$$
\mu_{n}\{(s, t)\}=\frac{\operatorname{dim} s}{n !}, \quad(s, t) \in B_{n} .
$$

This definition is motivated by the groupoid structure on the space of bitableaux. Namely, if we equip $B_{n}$ with the structure of a principal groupoid with diagonal $T_{n}$ as described in Sec. 5.2 for the infinite case, then $\mu_{n}$ is precisely the measure induced on the groupoid $B_{n}$ by the Plancherel measure $\mathrm{Pl}_{n}$ on $T_{n}$. In other words, the projection of $\mu_{n}$ to the first component $s$ is the Plancherel measure $\mathrm{Pl}_{n}$ on tableaux, and the conditional measure on the second component $t$ given a fixed first component $s$ is the counting measure. Clearly, the Plancherel measure on bitableaux is not normalized:

$$
\sum_{(s, t) \in B_{n}} \mu_{n}\{(s, t)\}=\sum_{(s, t) \in B_{n}} \frac{\operatorname{dim} t}{n !}=\sum_{\lambda \in B_{n}} \frac{\operatorname{dim}^{3} \lambda}{n !} \rightarrow \infty \quad \text { as } \quad n \rightarrow \infty .
$$

As we will see in Sec. 5.1, this measure is an analog of the "correct" Plancherel measure for the infinite symmetric group.

\subsection{The Fourier transform on the finite symmetric groups}

Definition 1. The Fourier transform on the finite symmetric group $\mathfrak{S}_{n}$ is the operator that maps a function $f \in \mathbb{C}\left[\mathfrak{S}_{n}\right]$ to a function $\widehat{f} \in \mathbb{C}\left(B_{n}\right)$ where

$$
\widehat{f}(s, t)=\sum_{w \in \mathfrak{S}_{n}} f(w)\left(\pi_{\lambda}(w) h_{s}, h_{t}\right), \quad(s, t) \in B_{n} .
$$

This formula can be understood as follows. Denote by End $V_{\lambda}$ the algebra of matrices in the space $V_{\lambda}$ of the representation $\pi_{\lambda}$, with distinguished basis consisting of the matrix elements of $\pi_{\lambda}$ with respect to the GelfandTsetlin basis. Note that this algebra, regarded as a subalgebra of $\mathbb{C}\left[\mathfrak{S}_{n}\right]$, is a minimal two-sided ideal. The representation $\pi_{\lambda}$ can be extended by linearity to the group algebra $\mathbb{C}\left[\mathfrak{S}_{n}\right]$. Thus for every function $f \in \mathbb{C}\left[\mathfrak{S}_{n}\right]$ we have the matrix $\pi_{\lambda}(f)=\sum_{w \in \mathfrak{S}_{n}} f(w) \pi_{\lambda}(w)$ from End $V_{\lambda}$. We can define the matrix Fourier transform of a function $f$ as

$$
\hat{f}(\lambda)=\pi_{\lambda}(f)=\sum_{w \in \mathfrak{S}_{n}} f(w) \pi_{\lambda}(w), \quad \lambda \in \mathbb{Y}_{n}
$$

Thus the matrix Fourier transform associates with a function $f \in \mathbb{C}\left[\mathfrak{S}_{n}\right]$ a function $\hat{f}$ on the set of diagrams $\mathbb{Y}_{n}$, with $\hat{f}(\lambda)$ taking values in End $V_{\lambda}$. The above-defined values $\hat{f}(s, t)$ are the matrix elements of $\hat{f}(\lambda)$ in the Gelfand-Tsetlin basis. This form of the Fourier transform will be called the tableau Fourier transform.

The inversion formula for the Fourier transform reads as

$$
f(w)=\sum_{(s, t) \in B_{n}} \hat{f}(s, t) \overline{\left(\pi^{\lambda}(w) h_{t}, h_{s}\right)} \mu_{n}(s, t), \quad w \in \mathfrak{S}_{n},
$$

or, in matrix form,

$$
f(w)=\sum_{\lambda \in \mathbb{Y}_{n}} \frac{\operatorname{dim} \lambda}{n !} \operatorname{tr}\left(\hat{f}(\lambda) \pi_{\lambda}^{*}(w)\right), \quad w \in \mathfrak{S}_{n}
$$

(where $*$ stands for the matrix conjugation).

The Fourier transform establishes an isometry between the spaces $L^{2}\left(\mathfrak{S}_{n}, m_{n}\right)$ and $L^{2}\left(B_{n}, \mu_{n}\right)$; namely, the following Plancherel formula holds:

$$
\sum_{w \in \mathfrak{S}_{n}} f(w) \overline{g(w)}=\sum_{(s, t) \in B_{n}} \widehat{f}(s, t) \overline{\widehat{g}(s, t)} \mu_{n}(s, t) .
$$

The Fourier transform sends convolution of functions on $\mathfrak{S}_{n}$ to matrix multiplication:

$$
\widehat{(f * g)}(\lambda)=\hat{f}(\lambda) \hat{g}(\lambda)
$$

or, in tableau form,

$$
\widehat{(f * g)}(s, t)=\sum_{r \sim s} \hat{f}(s, r) \hat{g}(r, t)
$$




\subsection{The Fourier transform of central functions}

Denote by $Z\left[\mathfrak{S}_{n}\right]$ the center of the group algebra of the symmetric group $\mathfrak{S}_{n}$, i.e., the set of central functions on $\mathfrak{S}_{n}$. For a central function $f \in Z\left[\mathfrak{S}_{n}\right]$, the operator $\pi_{\lambda}(f)=\sum_{w \in \mathfrak{S}_{n}} f(w) \pi_{\lambda}(w)$ is scalar on $V_{\lambda}$, thus the matrix Fourier transform of a central function $f$ takes the form

$$
\hat{f}(\lambda)=\left(\sum_{w \in \mathfrak{S}_{n}} f(w) \frac{\chi^{\lambda}(w)}{\operatorname{dim} \lambda}\right) E_{\lambda}, \quad t \in T(\lambda),
$$

where $E_{\lambda}$ is the identity operator in $V_{\lambda}$. In the tableau form, this means that the function $\hat{f}$ is supported by the diagonal $\left\{(t, t), t \in T_{n}\right\}$ and its value at a bitableau $(t, t) \in \lambda$ depends only on $\lambda$. Such functions on the space of bitableaux will be called central.

Thus the Fourier transform of a central function on $\mathfrak{S}_{n}$ can be identified with a function on the set $\mathbb{Y}_{n}$ of Young diagrams with $n$ cells. The inverse Fourier transform for central functions takes the form

$$
f(w)=\sum_{\lambda \in \mathbb{Y}_{n}} \chi^{\lambda}(w) \hat{f}(\lambda) \frac{\operatorname{dim} \lambda}{n !} ;
$$

in particular,

$$
f(e)=\sum_{\lambda \in \mathbb{Y}_{n}} \hat{f}(\lambda) \frac{\operatorname{dim}^{2} \lambda}{n !} ;
$$

the Plancherel formula in this case reads as

$$
\sum_{w \in \mathfrak{S}_{n}} f(w) \overline{g(w)}=\sum_{\lambda \in \mathbb{Y}_{n}} \hat{f}(\lambda) \overline{\hat{g}(\lambda)} \frac{\operatorname{dim}^{2} \lambda}{n !} .
$$

In particular, the Fourier transform of an irreducible character $\chi^{\lambda}$ is the function

$$
\widehat{\chi^{\lambda}}(\mu)=\left\{\begin{array}{lll}
0 & \text { if } \quad \mu \neq \lambda, \\
\frac{n !}{\operatorname{dim} \lambda} & \text { if } \quad \mu=\lambda .
\end{array}\right.
$$

Note (see [6]) that there is a canonical correspondence between central functions on the symmetric groups and symmetric functions.

\subsection{Gelfand-Tsetlin algebras and Young-Jucys-Murphy elements}

The key role in the inductive construction of the representation theory of the symmetric groups [15] (see also [16]) is played by the Gelfand-Tsetlin algebras and Young-Jucys-Murphy elements.

The Gelfand-Tsetlin algebra of the symmetric group $\mathfrak{S}_{n}$ is the subalgebra $\operatorname{GZ}(n) \subset \mathbb{C}\left[\mathfrak{S}_{n}\right]$ generated by the centers $Z\left[\mathfrak{S}_{1}\right], Z\left[\mathfrak{S}_{2}\right], \ldots, Z\left[\mathfrak{S}_{n}\right]$. This is a maximal commutative subalgebra of the group algebra $\mathbb{C}\left[\mathfrak{S}_{n}\right]$. Namely, this is the algebra of all operators diagonal in the Gelfand-Tsetlin basis.

Thus the Fourier transform of an element of the Gelfand-Tsetlin algebra is a function supported by the diagonal $T_{n}$.

The Young-Jucys-Murphy (YJM) elements are the following elements $X_{k} \in \mathbb{C}\left[\mathfrak{S}_{n}\right], k=1, \ldots, n$ :

$$
X_{k}=(1 k)+(2 k)+\ldots+(k-1 k)
$$

(as usual, $(i, j)$ stands for the transposition permuting $i$ and $j$ ). The YJM-elements form a multiplicative basis of the Gelfand-Tsetlin algebra GZ(n).

The matrix Fourier transform of a YJM-element $X_{k}$ sends a diagram $\lambda$ to the diagonal matrix from End $V_{\lambda}$ in which the diagonal element corresponding to a tableau $t \in \lambda$ is equal to the content ${ }^{1} c_{k}(t)$ of the cell of $t$ containing the element $k$. Thus the set of such diagonal matrices, with diagonal elements equal to the contents of tableaux, form a multiplicative basis in the algebra of all diagonal matrices, which is the Fourier transform of the Gelfand-Tsetlin algebra. The tableau Fourier transform of a YJM-element $X_{k}$ is the function on bitableaux given by the formula

$$
\widehat{X_{k}}(t, s)= \begin{cases}c_{k}(t) & \text { if } s=t, \\ 0 & \text { otherwise. }\end{cases}
$$

\footnotetext{
${ }^{1}$ Recall that the content of a cell $\square$ of a Young diagram lying at the intersection of the $i$ th row and $j$ th column is the number $c(\square)=i-j$. 


\section{The Infinite Symmetric GRoup $\mathfrak{S}_{\infty}$}

Necessary facts from the representation theory of the infinite symmetric group can be found, e.g., in [14].

Let $\mathfrak{S}_{\infty}=\cup_{n=1}^{\infty} \mathfrak{S}_{n}=\lim \mathfrak{S}_{n}$ be the infinite symmetric group with the fixed structure of inductive limit, and let $\mathbb{C}\left[\mathfrak{S}_{\infty}\right]$ be the group algebra of $\mathfrak{S}_{\infty}$.

Denote by $T=\lim T_{n}$ the space of infinite tableaux, i.e., the space of infinite paths in the Young graph.

The tail equivalence relation $\sim$ on $T$ is defined as follows: paths $s=\left(s_{1}, s_{2}, \ldots\right)$ and $t=\left(t_{1}, t_{2}, \ldots\right)$ are equivalent if and only if $s_{k}=t_{k}$ for sufficiently large $k$. In a similar way we define the $n$-equivalence relation on $T: s \sim_{n} t$ if and only if $s_{k}=t_{k}$ for $k \geq n$.

Consider the space $\mathcal{B}=\{(s, t): s, t \in T, s \sim t\}$ of infinite bitableaux (loops) with the topology of the inductive limit $\mathcal{B}=\lim \mathcal{B}_{n}$, where $\mathcal{B}_{n}=\left\{(s, t): s, t \in T, s \sim_{n} t\right\}$. Thus $\mathcal{B}$ is a separable totally disconnected locally compact space, and its diagonal $\mathcal{B}_{0}=\{(t, t), t \in T\}$ is homeomorphic to the space $T$ of paths.

The Fourier transform establishes a canonical isomorphism between the group algebra $\mathbb{C}\left[\mathfrak{S}_{\infty}\right]$ of the infinite symmetric group and the $*$-algebra $\mathbb{C}(\mathcal{B})$ of locally constant finitary functions on $\mathcal{B}$; multiplication in this algebra is defined as

$$
f g(s, t)=\sum_{r \sim t} f(s, r) g(r, t)
$$

and involution is given by the formula

$$
f^{*}(s, t)=\overline{f(t, s)}
$$

The Gelfand-Tsetlin algebra of the infinite symmetric group $\mathrm{GZ}(\infty)$ (i.e., the subalgebra in $\mathbb{C}\left[\mathfrak{S}_{\infty}\right]$ generated by all centers $\left.Z\left[\mathfrak{S}_{1}\right], Z\left[\mathfrak{S}_{2}\right], \ldots, Z\left[\mathfrak{S}_{n}\right], \ldots\right)$ goes to the commutative subalgebra of functions supported by the diagonal $\mathcal{B}_{0}$.

A measure $M$ on the space of paths $T$ is called central if the measure of each cylinder set $M\left(\left\{t:[t]_{n}=s\right\}\right)$, where $[t]_{n}$ is the initial segment of length $n$ of the path $t$ and $s$ is a fixed tableau from $T_{n}$, depends only on the shape of $t$. The cotransition probabilities $\operatorname{Prob}\left\{[t]_{n}=s \mid[t]_{n+1}=u\right\}$ are the same for all central measures and depend only on the graph.

The Plancherel measure on the space $T$ of infinite tableaux (i.e., infinite paths in the Young graph) is the Markov central measure $\mu$ with transition probabilities

$$
p_{\infty}(s, u)=\frac{\operatorname{dim} u}{(n+1) \operatorname{dim} s}, \quad s \in T_{n}, u \in T_{n+1},
$$

and initial distribution supported by the unique one-cell tableau. Thus the measure $\mu$ is defined on cylinder sets as follows:

$$
\mu\left(\left\{t \in T:[t]_{n}=s\right\}\right)=\frac{\operatorname{dim} s}{n !} .
$$

\section{The Plancherel expectation}

\subsection{Definition of the Plancherel expectation}

Let $i_{n}$ be the canonical embedding of $\mathfrak{S}_{n}$ into $\mathfrak{S}_{n+1}$. By the same letter we will denote the corresponding embedding of the group algebras $i_{n}: \mathbb{C}\left[\mathfrak{S}_{n}\right] \rightarrow \mathbb{C}\left[\mathfrak{S}_{n+1}\right]$. We will regard elements of the group algebra as measures on the group and, correspondingly, write them in the form $\sum_{w} f_{w} \delta_{w}$. Then the embedding $i_{n}$ takes the form

$$
\left(i_{n} f\right)_{u}=\left\{\begin{array}{ll}
f_{u} & \text { if } u \in \mathfrak{S}_{n}, \\
0 & \text { otherwise, }
\end{array} \quad f=\sum_{w \in \mathfrak{S}_{n+1}} f_{w} \delta_{w} \in \mathbb{C}\left[\mathfrak{S}_{n+1}\right], \quad u \in \mathfrak{S}_{n},\right.
$$

i.e., $i_{n}\left(\sum_{w \in \mathfrak{S}_{n+1}} f_{w} \delta_{w}\right)=\sum_{u \in \mathfrak{S}_{n+1}} f_{u} \delta_{u}$.

Consider the operator $p_{n}=i_{n}^{*}$ conjugate to $i_{n}$ (with respect to the scalar products in $\mathbb{C}\left[\mathfrak{S}_{n}\right]$ and $\mathbb{C}\left[\mathfrak{S}_{n+1}\right]$ determined by the measures $m_{n}$ and $m_{n+1}$, respectively). We will regard elements of the dual spaces to the group algebras (which, of course, are isomorphic to the group algebras themselves) as functions on groups and write them in functional notation. The projection $p_{n}: \mathbb{C}\left[\mathfrak{S}_{n+1}\right] \rightarrow \mathbb{C}\left[\mathfrak{S}_{n}\right]$ has the form

$$
\left(p_{n} f\right)(w)=f(w), \quad f \in \mathbb{C}\left[\mathfrak{S}_{n+1}\right], w \in \mathfrak{S}_{n} .
$$


Thus $p_{n}$ is the restriction to a subgroup. It is not difficult to check that $p_{n}$ is a positive conditional expectation ${ }^{2}$ from the algebra $\mathbb{C}\left[\mathfrak{S}_{n+1}\right]$ to the subalgebra $\mathbb{C}\left[\mathfrak{S}_{n}\right]$.

Recall (see [12]) that an arbitrary positive conditional expectation from $\mathbb{C}\left[\mathfrak{S}_{n+1}\right]$ to $\mathbb{C}\left[\mathfrak{S}_{n}\right]$ is determined by a collection of nonpositive numbers $a_{\mu \lambda}, \mu \in \mathbb{Y}_{n}, \lambda \in \mathbb{Y}_{n+1}$, such that $\sum_{\lambda: \mu \subset \lambda} a_{\mu \lambda}=1$ for every $\mu$. The abovedefined expectation $p_{n}$ corresponds to the Plancherel coefficients $a_{\mu \lambda}=p_{\infty}(\mu, \lambda)=\frac{\operatorname{dim} \lambda}{(n+1) \operatorname{dim} \mu}$, thus it is natural to call it the Plancherel conditional expectation.

The inductive limit of the group algebras $\mathbb{C}\left[\mathfrak{S}_{n}\right]$ with respect to the embeddings $i_{n}$ is the group algebra of the infinite symmetric group:

$$
\underset{\lim }{\longrightarrow}\left(\mathbb{C}\left[\mathfrak{S}_{n}\right], i_{n}\right)=\mathbb{C}\left[\mathfrak{S}_{\infty}\right]
$$

The projective limit of the group algebras $\mathbb{C}\left[\mathfrak{S}_{n}\right]$ (regarded as spaces of functions on the groups) with respect to the conditional expectations $p_{n}$ is the space of arbitrary functions on the infinite symmetric group:

$$
\lim _{\longleftarrow}\left(\mathbb{C}\left[\mathfrak{S}_{n}\right], p_{n}\right)=\mathbb{F}\left(\mathfrak{S}_{\infty}\right) .
$$

\subsection{The Fourier transform of the Plancherel conditional expectation}

An easy calculation shows that the Fourier transform sends the embedding $i_{n}$ to the operator (for simplicity, we will denote it by the same letter) $i_{n}: \mathbb{C}\left(B_{n}\right) \rightarrow \mathbb{C}\left(B_{n+1}\right)$, where for $f \in \mathbb{C}\left(B_{n}\right),(s, t) \in B_{n+1}$,

$$
\left(i_{n} f\right)(s, t)= \begin{cases}f\left([s]_{n},[t]_{n}\right) & \text { if } s \sim_{n} t \\ 0 & \text { otherwise. }\end{cases}
$$

The Plancherel conditional expectation goes to the operator $p_{n}: \mathbb{C}\left(B_{n+1}\right) \rightarrow \mathbb{C}\left(B_{n}\right)$, where

$$
\left(p_{n} f\right)(s, t)=\sum_{(u, v) \in B_{n+1}:(s, t) \nearrow(u, v)} p_{\infty}(s, u) f(u, v), \quad f \in \mathbb{C}\left(B_{n+1}\right),(s, t) \in B_{n} ;
$$

here $p_{\infty}(s, u)$ is the Plancherel transition probability $(6)$, and the notation $(s, t) \nearrow(u, v)$ means that the pair of paths $(s, t)$ is obtained from the pair $(u, v)$ by removing the last vertex.

The inductive limit of the spaces $\mathbb{C}\left(B_{n}\right)$ with respect to the operators $i_{n}$ is the space of finitary functions on the space of infinite bitableaux $\mathcal{B}$ :

$$
\underset{\lim }{\longrightarrow}\left(\mathbb{C}\left(B_{n}\right), i_{n}\right)=\mathbb{C}_{\text {fin }}(\mathcal{B}) .
$$

Our nearest goal is to describe the projective limit of the spaces $\mathbb{C}\left(B_{n}\right)$ with respect to the Plancherel conditional expectations $p_{n}$.

\section{The full Plancherel measure and the projective limit of the spaces $\mathbb{C}\left(B_{n}\right)$ With Respect to the Plancherel Conditional expectations}

\subsection{The full Plancherel measure}

Traditionally, Plancherel measure associated with the infinite symmetric group is understood as the Plancherel measure on the space of infinite tableaux $T$ introduced in Sec. 3. However, the "true" Plancherel measure for the infinite symmetric group $\mathfrak{S}_{\infty}$ is a measure in the space of bitableaux $\mathcal{B}$, which we introduce in this section. For this measure, there is a Plancherel theorem, i.e., the Fourier transform establishes an isometry between the space of square integrable functions on $\mathfrak{S}_{\infty}$ with respect to the counting measure and the space of square integrable functions on $\mathcal{B}$ with respect to this Plancherel measure (Theorem 2).

Given a tableau $t \in T$, denote by $G^{t}=\{(t, \cdot) \in \mathcal{B}\}$ the countable set of bitableaux with the first component equal to $t$. Let $\lambda^{t}$ be the counting measure on the set $G^{t}$.

Definition 2. The full Plancherel measure on the space of bitableaux $\mathcal{B}$ is the $\sigma$-finite measure

$$
\tilde{\mu}=\int_{T} \lambda^{t} d \mu(t) .
$$

\footnotetext{
${ }^{2}$ Concerning the notion of conditional expectation from a $C^{*}$-algebra to its $C^{*}$-subalgebra, see, e.g., [9]; note that in the classical version, one considers only positive expectations; concerning nonpositive expectations, see [12] and Sec. 7.1.
} 
In other words, this is the measure whose projection to the first component coincides with the Plancherel measure $\mu$ on the space of tableaux $T$ and the conditional distribution on the second component for a fixed first component is the counting measure.

In Sec 5.2, we will explain that this definition is directly related to the groupoid structure on the space of bitableaux, and it should be understood precisely in this way. On the other hand, in some cases it is convenient to use the following explicit description of the full Plancherel measure.

Let $B=\cup_{n=1}^{\infty} B_{n}$ be the disjoint union of the sets of finite loops. We say that a finite bitableau $(a, b) \in B$ is proper if $(a, b) \in B_{n}$ and $a \nsim_{n-1} b$ (i.e., $\left.\left([a]_{n-1},[b]_{n-1}\right) \notin B_{n-1}\right)$. By definition, the unique element of the first level is a proper bitableau. Denote by $B_{\text {prop }} \subset B$ the set of all proper bitableaux.

There is a natural mapping $P: \mathcal{B} \rightarrow B_{\text {prop }}$, where $P(s, t)=\left([s]_{n},[t]_{n}\right)$ if $s \sim_{n} t$ but $s \nsim_{n-1} t$. Moreover, if $a, b \in T(\lambda), \lambda \in \mathbb{Y}_{n}$, then the set $T_{(a, b)}=P^{-1}(a, b)$ can be naturally identified with the set $T_{\lambda}$ of infinite paths in the Young graph starting from the vertex $\lambda$. (In particular, the space $T_{(1,1)}$, where $(1,1)$ is the unique loop of the first level, is naturally identified with the space $T$ of all infinite paths in the Young graph.) Thus we have a natural decomposition

$$
\mathcal{B}=\bigcup_{(a, b) \in B_{\text {prop }}} T_{(a, b)}
$$

On the set $T_{(a, b)} \simeq T_{\lambda}$ we have the distribution $\mu_{(a, b)}=\mu_{\lambda}$ induced by the Plancherel measure $\mu$ on $T$, which is just the law of the random walk on $T$ starting from the vertex $\lambda$ and having the Plancherel transition probabilities (6). The full Plancherel measure on the space of bitableaux $\mathcal{B}$ can be represented in the form

$$
\tilde{\mu}=\sum_{(a, b) \in B_{\text {prop }}} \frac{\operatorname{dim} a}{n !} \mu_{(a, b)}, \quad(a, b) \in B_{n} .
$$

\subsection{Groupoid structure on the space of bitableaux}

It is convenient to describe the space of bitableaux and measures on it in terms of groupoid theory (see, e.g., [10]).

We can regard the space of bitableaux $\mathcal{B}=\{(s, t): s, t \in T, s \sim t\}$ as the principal groupoid generated by the tail equivalence relation on paths. Namely, pairs $(s, t)$ and $(u, v)$ are composable if and only if $u=t$, and $(s, t) \cdot(t, v)=(s, v)$. The mappings $r$ and $d$ are given by the formulas $r(s, t)=(s, s)$ and $d(s, t)=(t, t)$, respectively. The unit space can be identified with the diagonal $\mathcal{B}_{0}=\{(s, s), s \in T\}$, i.e., with the space $T$ of infinite tableaux. The set $G^{s}=r^{-1}(s)$ for $s \in T$ is $G^{s}=\{(s, t), t \sim s\}$ (it is a countable set). The left Haar system on the principal groupoid $B$ consists of the counting measures $\lambda^{t}$ on the sets $G^{t}$. Consider the Plancherel measure $\mu$ on the diagonal $T$. The measure $\int_{T} \lambda^{t} d \mu(t)$ on the groupoid $\mathcal{B}$ generated by $\mu$ is precisely the full Plancherel measure $\tilde{\mu}$.

\subsection{The projective limit of the spaces $\mathbb{C}\left(B_{n}\right)$ with respect to the Plancherel conditional expecta- tions}

Theorem 1. The projective limit of the spaces $\mathbb{C}\left(B_{n}\right)$ with respect to the Plancherel conditional expectations $p_{n}$ is the space

$$
\lim _{\longleftarrow}\left(\mathbb{C}\left(B_{n}\right), p_{n}\right)=\operatorname{Mart}(\mathcal{B}, \tilde{\mu})
$$

of martingales on the space of infinite bitableaux $\mathcal{B}$ with respect to the full Plancherel measure $\tilde{\mu}$.

The space of martingales $\operatorname{Mart}(\mathcal{B}, \tilde{\mu})$ with respect to the $\sigma$-finite measure $\tilde{\mu}$ is understood as the space of collections $\left\{\phi_{(a, b)}\right\}_{(a, b) \in B_{\text {prop }}}$, where $\phi_{(a, b)}$ is a martingale on $T_{(a, b)}$ with respect to the measure $\mu_{(a, b)}$.

Proof. Let $\mathfrak{A}$ be the Borel $\sigma$-algebra in the space $\mathcal{B}$. Denote by $\mathfrak{A}_{n}$ its $\sigma$-subalgebra generated by functions supported by $\mathcal{B}_{n}$ and depending only on the first $n$ coordinates of a bitableau (the set of such functions is naturally identified with $\left.\mathbb{C}\left(B_{n}\right)\right)$. The theorem follows from the fact that, as can easily be seen, the right-hand side of formula (7) describes the conditional expectation of a function $f$ with respect to the $\sigma$-algebra $\mathfrak{A}_{n}$.

Thus we obtain the following definition of the Fourier transform on the infinite symmetric group $\mathfrak{S}_{\infty}$.

Definition 3. The Fourier transform of an arbitrary function $f$ on the infinite symmetric group $\mathfrak{S}_{\infty}$ is the martingale $\left(\phi_{1}, \phi_{2}, \ldots\right)$ on the space of infinite bitableaux $\mathcal{B}$ with respect to the full Plancherel measure $\tilde{\mu}$, where $\phi_{n}=\widehat{f_{n}}$ is the Fourier transform of the restriction $f_{n}$ of the function $f$ to the finite symmetric group $\mathfrak{S}_{n}$. 


\section{The Fourier transform of Different Classes of FunCtions on $\mathfrak{S}_{\infty}$}

\subsection{Square integrable functions}

Consider the counting measure $m$ on the infinite symmetric group $\mathfrak{S}_{\infty}$.

Theorem 2. The Fourier transform is an isometry of the space $l_{m}^{2}\left(\mathfrak{S}_{\infty}\right)$ of square integrable functions on the infinite symmetric group $\mathfrak{S}_{\infty}$ with respect to the counting measure and the space $L^{2}(\mathcal{B}, \tilde{\mu})$ of square integrable functions on the space of infinite bitableaux $\mathcal{B}$ with respect to the full Plancherel measure $\tilde{\mu}$. The Plancherel formula takes the form

$$
\sum_{w \in \mathfrak{S}_{\infty}} f(w) \bar{g}(w)=\int_{\mathcal{B}} \hat{f}(s, t) \hat{g}(s, t) d \tilde{\mu}(s, t), \quad f, g \in l_{m}^{2}\left(\mathfrak{S}_{\infty}\right)
$$

Proof. The Fourier transform of a square integrable function on $\mathfrak{S}_{\infty}$ is a square integrable martingale with respect to the full Plancherel measure (since the Plancherel conditional expectations $p_{n}$ accord with the Hilbert structures in the group algebras), which, according to martingale theory, in turn corresponds to a square integrable function on $\mathcal{B}$ with respect to the full Plancherel measure.

\subsection{The Gelfand-Tsetlin space}

As observed above, the Fourier transform of an element of the Gelfand-Tsetlin algebra GZ $(\infty)$ of the infinite symmetric group is a function supported by the diagonal $T$.

Clearly, the Plancherel conditional expectation (as well as any other conditional expectation from $\mathbb{C}\left[\mathfrak{S}_{n+1}\right]$ to $\left.\mathbb{C}\left[\mathfrak{S}_{n}\right]\right)$ sends the Gelfand-Tsetlin algebra GZ $(n+1)$ to $\mathrm{GZ}(n)$, thus we can consider the space GZ $=\lim ^{\mathrm{GZ}}(n)$. It is no longer an algebra, but is a left and right $\mathbb{C}\left[\mathfrak{S}_{\infty}\right]$-bimodule. We will call it the Gelfand-Tsetlin space of the infinite symmetric group. It follows from above that the Fourier transform of an element from GZ is a martingale on the space of paths $T$ with respect to the Plancherel measure $\mu$.

\subsection{Central functions}

The Fourier transform of a central function on $\mathfrak{S}_{\infty}$ is a central martingale $\left(f^{1}, f^{2}, \ldots\right)$ on the space of tableaux $T$ with respect to the Plancherel measure, which means that all functions $f^{k}$ are central in the sense of Sec. 2.4 (i.e., they are supported by the diagonal and depend only on the shape of tableaux).

For instance, let $f$ be the characteristic function of a conjugacy class in $\mathfrak{S}_{\infty}$. This means that there exists a diagram $\rho \in \mathbb{Y}_{k}$ without rows of length 1 such that the restriction of $f$ to $\mathfrak{S}_{n}$ for $n \geq k$ is the characteristic function of the conjugacy class $C_{\rho_{n}}$ corresponding to the diagram $\rho_{n}=\rho \cup 1^{n-k} \in \mathbb{Y}_{n}$. Then the Fourier transform of the function $f=f_{\rho}$ is the central martingale $\left(\phi_{1}, \phi_{2}, \ldots\right)$, where

$$
\phi_{n}(s, t)= \begin{cases}\frac{\chi^{\lambda}\left(\rho_{n}\right)}{\operatorname{dim} \lambda}\left|C_{\rho_{n}}\right| & \text { if } s=t \text { and }[t]_{n} \in \lambda, \\ 0 & \text { otherwise. }\end{cases}
$$

\subsection{Positive definite functions}

The Fourier transform of a positive definite function on $\mathfrak{S}_{\infty}$ is a martingale $\left(f^{1}, f^{2}, \ldots\right)$ such that

$$
\sum_{(s, t) \in B_{n}} f^{n}(s, t) h(s) \bar{h}(t) \frac{\operatorname{dim} s}{n !} \geq 0 \quad \text { for every } \quad n \in \mathbb{N}, \quad \text { for every } \quad h \in \mathbb{C}\left(T_{n}\right)
$$

In particular, the restriction of a positive definite martingale to the diagonal $\{(t, t)\} \simeq T$ is a positive martingale on $T$. Note that there is a natural one-to-one correspondence between positive martingales on $T$ and (positive) measures on $T=\lim _{\longleftarrow} T_{n}$. Namely, $\phi=\left(\phi_{1}, \phi_{2}, \ldots\right) \leftrightarrow M^{\phi}=\lim _{\longleftarrow} M_{n}^{\phi}$, where

$$
M_{n}^{\phi}(s):=M^{\phi}\left(\left\{t:[t]_{n}=s\right\}\right)=\phi_{n}(s) \frac{\operatorname{dim} s}{n !}, \quad s \in T_{n} .
$$

Thus the restriction of a positive definite martingale to the diagonal (i.e., the Fourier transform of the projection of the original central function to the Gelfand-Tsetlin space) is a (positive) measure on the space of paths $T$. 


\subsection{Characters}

In particular, if $f$ is a character, i.e., a central positive definite normalized $(f(e)=1)$ function on $\mathfrak{S}_{\infty}$, then its Fourier transform is a positive probability central measure on $T$ (in view of (4), a central function is normalized if and only if the corresponding measure is normalized). Thus we obtain the classical correspondence (see, e.g., [14] or [4]) $\chi \leftrightarrow M$ between characters of the infinite symmetric group and central probability measures on the space of bitableaux, which is given by the formula

$$
\chi_{n}=\sum_{\lambda \in \mathbb{Y}_{n}} M^{(n)}(\lambda) \frac{\chi^{\lambda}}{\operatorname{dim} \lambda},
$$

where $\chi_{n}=\left.\chi\right|_{\mathfrak{S}_{n}}$ and $M^{(n)}(\lambda)=M\left(\left\{t:[t]_{n} \in \lambda\right\}\right)$.

\subsection{Examples}

1. The Fourier transform of the $\delta$-function $\delta_{g}$ at an element $g \in \mathfrak{S}_{\infty}$ is the function ${ }^{3}$

$$
\hat{\delta}_{g}(s, t)= \begin{cases}\left(\pi^{\lambda}(g) h_{[t]_{n}}, h_{[s]_{n}}\right), & s \sim_{n} t, \\ 0 & \text { otherwise, }\end{cases}
$$

where $g \in \mathfrak{S}_{n}$ and $\lambda$ is the shape of the tableaux $[s]_{n},[t]_{n}$.

In particular, $\hat{\delta}_{e}(s, t)=\delta_{s t}$ is the characteristic function of the diagonal.

2. Using the Young orthogonal form (see, e.g., [1]), we can write the Fourier transforms of the Coxeter generators $\sigma_{k}=(k, k+1)$ in a more explicit form. Namely,

$$
\hat{\sigma}_{k}(s, t)= \begin{cases}\delta_{t s} & \text { if } k \text { and } k+1 \text { are in the same row of } t, \\ -\delta_{t s} & \text { if } k \text { and } k+1 \text { are in the same column of } t, \\ \rho_{1} \delta_{t s}+\rho_{2} \delta_{\sigma_{k} t, s} & \text { otherwise, }\end{cases}
$$

where

$$
\rho_{1}=\frac{1}{c_{k+1}(t)-c_{k}(t)}, \quad \rho_{2}=\sqrt{1-\rho_{1}^{2}},
$$

and the tableau $\sigma_{k} t$ is obtained from $t$ by permuting the elements $k$ and $k+1$.

3. As noted above, the Fourier transform of a Young-Jucys-Murphy element is the function

$$
\hat{X}_{k}(s, t)= \begin{cases}c_{k}(t), & s=t, \\ 0, & s \neq t,\end{cases}
$$

where $c_{k}(t)$ is the content of the cell of $t$ that contains $k$.

4. One can use the Fourier transform and the inverse Fourier transform to transfer the action of operators from the group algebra to the space of functions on bitableaux and vice versa. For example, consider the operator that acts on tableaux (and hence on bitableaux) by reflecting with respect to the main diagonal (more exactly, the induced operator on functions). It is easy to see that the corresponding operator on the group algebra is the operator of multiplication by the sign $\varepsilon(g)$ of a permutation, i.e., $a(g) \mapsto \varepsilon(g) a(g)$.

\section{OTHER CONDITIONAL EXPECTATIONS}

\subsection{Virtual projection}

Let $\pi_{n}: \mathfrak{S}_{n+1} \rightarrow \mathfrak{S}_{n}$ be the virtual projection, which associates with a permutation $w \in \mathfrak{S}_{n+1}$ its derivative permutation, i.e., the permutation obtained by deleting the element $n+1$ from the corresponding cycle of $w$. By the same letter we will denote the extension of the virtual projection to the group algebras by linearity, i.e., the projection $\pi_{n}: \mathbb{C}\left[\mathfrak{S}_{n+1}\right] \rightarrow \mathbb{C}\left[\mathfrak{S}_{n}\right]$ given by the formula (elements of the group algebra are regarded as measures)

$$
\left(\pi_{n} f\right)_{u}=\sum_{\substack{w \in \mathfrak{G}_{n+1} \\ \pi_{n} w=u}} f_{w}, \quad f=\sum_{w \in \mathfrak{S}_{n+1}} f_{w} \delta_{w}, \quad u \in \mathfrak{S}_{n} .
$$

This projection is a generalized (i.e., nonpositive) conditional expectation from $\mathbb{C}\left[\mathfrak{S}_{n+1}\right]$ to $\mathbb{C}\left[\mathfrak{S}_{n}\right]$. Let us recall the corresponding definition (see [12]). Let $A$ be a $C^{*}$-algebra with identity over $\mathbb{C}$ and $B$ be its $C^{*}$ subalgebra. A linear operator $P: A \longrightarrow B$ is called a conditional expectation, or simply expectation, from the algebra $A$ to the subalgebra $B$ if (1) $P(b)=b$ and $P\left(b_{1} a b_{2}\right)=b_{1} P(a) b_{2}$ for every $a \in A$ and $b, b_{1}, b_{2} \in B ;(2)$ $P\left(a^{*}\right)=a^{*}, P 1=1$; (3) $P \geq 0$, which means that for every $a \in A$ the element $P\left(a a^{*}\right)$ is positive, i.e., belongs to the real cone in $B$ generated by elements of the form $b b^{*}$. If only conditions (1) and (2) hold, then $P$ is called a generalized expectation.

\footnotetext{
${ }^{3}$ Recall that $h_{t}$ stands for the element of the Gelfand-Tsetlin basis corresponding to the tableau $t$.
} 
Lemma 1. For $n \geq 4$, the virtual projection is the only conditional expectation from $\mathbb{C}\left[\mathfrak{S}_{n+1}\right]$ to $\mathbb{C}\left[\mathfrak{S}_{n}\right]$ satisfying the following condition: an element of the group $\mathfrak{S}_{n+1}$ (regarded as an element of the group algebra) goes to an element of the group $\mathfrak{S}_{n}$.

The conjugate (with respect to the counting measures on $\mathfrak{S}_{n+1}$ and $\mathfrak{S}_{n}$ ) operator $\pi_{n}^{*}: \mathbb{C}\left[\mathfrak{S}_{n}\right] \rightarrow \mathbb{C}\left[\mathfrak{S}_{n+1}\right]$ takes the form (in the conjugate space, elements of the group algebra are regarded as functions)

$$
\pi_{n}^{*} f(w)=f\left(\pi_{n} w\right), \quad f \in \mathbb{C}\left[\mathfrak{S}_{n}\right], \quad w \in \mathfrak{S}_{n+1} .
$$

The projective limit of the symmetric groups with respect to the virtual projections $\pi_{n}$ is called the space of virtual permutations $\mathfrak{S}^{\infty}$ :

$$
\mathfrak{S}^{\infty}=\lim _{\longleftarrow}\left(\mathfrak{S}_{n}, \pi_{n}\right) .
$$

This space was introduced in [4] (see also a detailed version [5]).

The inductive limit of the group algebras $\mathbb{C}\left[\mathfrak{S}_{n}\right]$ with respect to the operators $\pi_{n}^{*}$ is the space of finitary functions on the space of virtual permutations:

$$
\underset{\lim }{\longrightarrow}\left(\mathbb{C}\left[\mathfrak{S}_{n}\right], \pi_{n}^{*}\right)=\mathbb{C}_{\text {fin }}\left(\mathfrak{S}^{\infty}\right)
$$

The projective limit of the group algebras with respect to the projections $\pi_{n}$ is the space of Borel measures on the space of virtual permutations:

$$
\underset{\lim }{\longrightarrow}\left(\mathbb{C}\left[\mathfrak{S}_{n}\right], \pi_{n}\right)=\mathcal{M}\left(\mathfrak{S}^{\infty}\right) .
$$

The Fourier transform sends the virtual projection and the conjugate operator to the operators

$$
\left(\pi_{n} f\right)(s, t)=\sum_{(u, v) \in B_{n+1}:(s, t) \nearrow(u, v)} p_{\infty}(s, u)\left(c_{n+1}(u)+1\right) f(u, v), \quad f \in \mathbb{C}\left(B_{n+1}\right), \quad(s, t) \in B_{n},
$$

and

respectively.

$$
\left(\pi_{n}^{*} f\right)(s, t)=\left\{\begin{array}{ll}
f\left([s]_{n},[t]_{n}\right)\left(c_{n+1}(t)+1\right) & \text { if } s \sim_{n} t, \\
0 & \text { otherwise, }
\end{array} \quad f \in \mathbb{C}\left(B_{n}\right), \quad(s, t) \in B_{n+1},\right.
$$

The inconvenience of the projection $\pi_{n}$ is that it does not accord with the Hilbert structures in the group algebras determined by the counting measures on the symmetric groups. To overcome this difficulty, one should consider the normalized projections $\tilde{\pi}_{n}=\frac{1}{\sqrt{n+1}} \pi_{n}$, i.e.,

$$
\begin{aligned}
\left(\pi_{n} f\right)_{u} & =\frac{1}{\sqrt{n+1}} \sum_{\substack{w \in \mathfrak{S}_{n+1}+1 \\
\pi_{n} w=u}} f_{w}, & f=\sum_{w \in \mathfrak{S}_{n+1}} f_{w} w, \quad u \in \mathfrak{S}_{n}, \\
\left(\pi_{n} f\right)(s, t) & =\frac{1}{\sqrt{n+1}} \sum_{(u, v) \in B_{n+1}:(s, t) \nearrow(u, v)} p_{\infty}(s, u)\left(c_{n+1}(u)+1\right) f(u, v), & f \in \mathbb{C}\left(B_{n+1}\right), \quad(s, t) \in B_{n} .
\end{aligned}
$$

7.2. $z$-Projections

Let $z \in \mathbb{C} \cup\{\infty\}, t=|z|^{2}$.

The $z$-projection $p_{n}^{z}: \mathbb{C}\left[\mathfrak{S}_{n+1}\right] \rightarrow \mathbb{C}\left[\mathfrak{S}_{n}\right]$ is the following linear combination of the Plancherel and virtual projections:

$$
p_{n}^{z}=\frac{1}{\sqrt{n+t}}\left((z-1) p_{n}+\pi_{n}\right)
$$

An explicit formula takes the form

$$
\left(p_{n}^{z} f\right)(u)=\frac{1}{\sqrt{n+t}}\left(\sum_{\substack{w \in \mathfrak{S}_{n+1} \\ \pi_{n} w=u}} f(w)+(z-1) f(u) 1_{u \in \mathfrak{S}_{n}}\right), \quad f \in \mathbb{C}\left[\mathfrak{S}_{n+1}\right], \quad u \in \mathfrak{S}_{n} .
$$

The Fourier transform sends the $z$-projection to the operator

$$
\left(\pi_{n} f\right)(s, t)=\frac{1}{\sqrt{n+t}} \sum_{(u, v) \in B_{n+1}:(s, t) \nearrow(u, v)} p_{\infty}(s, u)\left(c_{n+1}(u)+z\right) f(u, v), \quad f \in \mathbb{C}\left(B_{n+1}\right), \quad(s, t) \in B_{n} .
$$

The virtual and Plancherel projections are particular cases of the $z$-projection, corresponding to $z=1$ and $z=\infty$, respectively. 
Lemma 2. For $n \geq 4$, the $z$-projections exhaust all conditional expectations from $\mathbb{C}\left[\mathfrak{S}_{n+1}\right]$ to $\mathbb{C}\left[\mathfrak{S}_{n}\right]$ satisfying the following condition: an element of the group $\mathfrak{S}_{n+1}$ goes to an element of the group $\mathfrak{S}_{n}$ with, possibly, some coefficient.

In particular, the Plancherel projection sends an element of the group to an element of the group or 0.

Lemma 3. For every function $f \in \mathbb{C}\left[\mathfrak{S}_{n}\right]$,

$$
\left(p_{1}^{z} p_{2}^{z} \ldots p_{n-1}^{z} p_{n}^{z} f\right)(w)=\left\langle f, z^{[w]}\right\rangle
$$

where $\langle\cdot, \cdot\rangle$ stands for the scalar product in $\mathbb{C}\left[\mathfrak{S}_{n}\right]$ determined by the counting measure on $\mathfrak{S}_{n}$, and $[w]$ denotes the number of cycles of a permutation $w \in \mathfrak{S}_{n}$.

In particular, for the Plancherel expectation we have

$$
\left(p_{1} p_{2} \ldots p_{n-1} p_{n} f\right)(w)=\left\langle f, \delta_{e}\right\rangle=f(e),
$$

and for the virtual projection,

$$
\left(\pi_{1} \pi_{2} \ldots \pi_{n-1} \pi_{n} f\right)(w)=\langle f, 1\rangle=\sum_{w \in \mathfrak{S}_{n}} f(w)
$$

Supported by the CRDF project RUM1-2622-ST-04, RFBR project 05-01-00899, and INTAS project 0351-5018, as well as by the President of Russian Federation Grant for Support of Leading Scientific Schools NSh.-2251.2003.1.

Translated by N. V. Tsilevich.

\section{REFERENCES}

1. G. James, The Representation Theory of the Symmetric Group, Springer, Berlin (1978).

2. G. James and A. Kerber, The Representation Theory of the Symmetric Group, Addison-Wesley, Reading, Massachusetts (1981).

3. E. Hewitt and K. Ross, Abstract Harmonic Analysis, Vol. 1, Academic Press, New York; Springer-Verlag, Berlin-Gottingen-Heidelberg, 1963; Vol. 2, Springer-Verlag, New York-Berlin (1970).

4. S. Kerov, G. Olshanski, and A. Vershik, "Harmonic analysis on the infinite symmetric group. A deformation of the regular representation," C. R. Acad. Sci. Paris Ser. I. Math, 316, No. 8, 773-778 (1993).

5. S. Kerov, G. Olshanski, and A. Vershik, "Harmonic analysis on the infinite symmetric group," Invent. Math., 158, No. 3, 551-642 (2004).

6. I. Macdonald, Symmetric Functions and Hall Polynomials, 2nd edition, Clarendon Press, Oxford (1995).

7. A. Yu. Okounkov, "Thoma's theorem and representations of an infinite bisymmetric group," Funct. Anal. Appl., 28, No. 2, 100-107 (1994).

8. G. I. Olshanski, "Unitary representations of $(G, K)$-pairs that are connected with the infinite symmetric group $S(\infty)$," Leningrad Math. J., 1, No. 4, 983-1014 (1990).

9. G. K. Pedersen, $C^{*}$-Algebras and Their Automorphism Groups, Academic Press, London-New York (1979).

10. J. Renault, A Groupoid Approach to $C^{*}$-Algebras, Lect. Notes Math., 793, Springer-Verlag, Berlin-Heidelberg-New York (1980).

11. R. Stanley, Enumerative Combinatorics, Vol. 2, Cambridge Univ. Press, Cambridge (1999).

12. A. Vershik, "Gelfand-Tsetlin algebras, expectations, inverse limits, Fourier analysis," in: Unity of Mathematics. In Honor of the Ninetieth Birthday of I. M. Gelfand, Birkhäuser (to appear).

13. A. M. Vershik and S. V. Kerov, "Asymptotic theory of characters of the symmetric group," Funct. Anal. Appl., 15, 246-255 (1982).

14. A. M. Vershik and S. V. Kerov, "The Grothendieck group of infinite symmetric group and symmetric functions (with the elements of the theory of $\mathrm{K}_{0}$-functor of AF-algebras)," in: Representation of Lie Groups and Related Topics, A. M. Vershik and D. P. Zhelobenko (eds.), Gordon and Breach Sci. Publ. (1990), pp. 39-118.

15. A. M. Vershik and A. Yu. Okounkov, "A new approach to the representation theory of the symmetric groups. II," Zap. Nauchn. Semin. POMI, 307, 57-98 (2004).

16. A. M. Vershik and A. Yu. Okounkov, "A new approach to the representation theory of the symmetric groups. II," Addendum to the Russian translation of the book W. Fulton, Young Tableaux, Moscow (2004). 